\title{
EchoGéo
}

44 | 2018

Les valorisations territoriales et touristiques du street art

\section{Temporalités du street art et image des territoires en mutation}

Production et valorisation du street art dans la Zone d'Aménagement Concerté Paris Rive Gauche

\section{Clotilde Kulmann}

\section{(2) OpenEdition}

\section{Journals}

Electronic version

URL: https://journals.openedition.org/echogeo/15467

DOI: $10.4000 /$ echogeo. 15467

ISSN: 1963-1197

Publisher

Pôle de recherche pour l'organisation et la diffusion de l'information géographique (CNRS UMR 8586)

\section{Electronic reference}

Clotilde Kulmann, "Temporalités du street art et image des territoires en mutation", EchoGéo [Online], 44 | 2018, Online since 31 July 2018, connection on 31 July 2021. URL: http://

journals.openedition.org/echogeo/15467 ; DOI: https://doi.org/10.4000/echogeo.15467

This text was automatically generated on 31 July 2021.

EchoGéo est mis à disposition selon les termes de la licence Creative Commons Attribution - Pas d'Utilisation Commerciale - Pas de Modification 4.0 International (CC BY-NC-ND) 


\title{
Temporalités du street art et image des territoires en mutation
}

\author{
Production et valorisation du street art dans la Zone d'Aménagement \\ Concerté Paris Rive Gauche
}

\section{Clotilde Kulmann}

1 Depuis la fin des années 2000, le nombre d'interventions de street art commandées dans la Zone d'Aménagement Concerté ${ }^{1}$ (ZAC) Paris Rive Gauche du $13^{\mathrm{e}}$ arrondissement de Paris par les professionnels de l'aménagement (sociétés d'aménagement, promoteurs, entreprises de travaux, bailleurs sociaux, maîtres d'œuvre, etc.), les autorités publiques (la municipalité $\mathrm{du} 13^{\mathrm{e}}$ arrondissement, la Ville de Paris, etc.), et les institutions culturelles (Nuit Blanche, musées, etc.) ne cesse d'augmenter. Plus d'une trentaine d'œuvres ont été produites à partir de médias variés, tels que la peinture, le collage et la mosaïque, sur différents types de supports comme les murs, les trottoirs et les mobiliers urbains, dans des espaces déjà ouverts aux usagers, des emprises de chantier, en extérieur et à l'intérieur de bâtiments vacants.

2 Ces créations visent à contribuer à la formation d'une image positive et attractive de la ZAC qui, engagée en 1991, reste encore peu fréquentée par les usagers qui n'y résident ou n'y travaillent pas. Ce manque d'image, voire d'appropriation collective, résulte notamment des choix techniques effectués pour régénérer le secteur. Située à l'emplacement d'une ancienne friche industrielle et ferroviaire isolée du reste de l'arrondissement par des voies ferrées, sa connexion à son environnement spatial s'est traduite par la couverture des rails, effaçant ainsi une partie des traces de son passé, et par la création d'espaces publics artificiels et d'infrastructures neuves, encore non patinées par le temps. L'un des objectifs de l'opération est donc d'ancrer symboliquement la ZAC dans Paris, d'y développer une histoire, de susciter l'intérêt des visiteurs et favoriser son rayonnement métropolitain, d'autant que sa situation en trait d'union entre le centre parisien et Seine Amont lui confère une position stratégique à l'échelle du projet du Grand Paris. 
Illustration 1 - Situation de la ZAC Paris Rive Gauche dans le 13e arrondissement de Paris

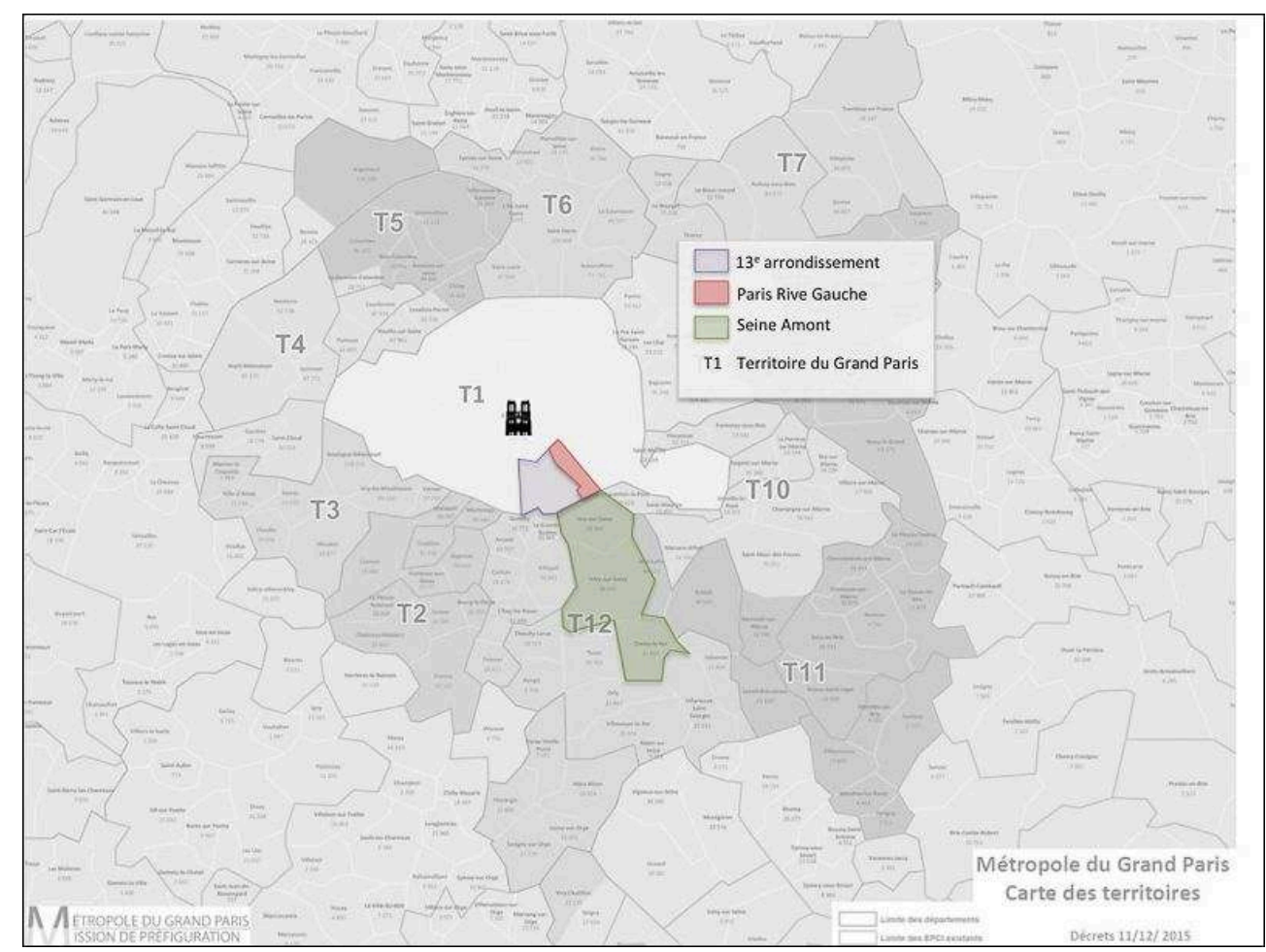

Ces enjeux d'image ne peuvent être appréhendés sans tenir compte de l'inscription de l'opération dans un temps long et incertain ${ }^{2}$ et des multiples temporalités qui la composent. Étant donné son importante envergure spatiale (cent-trente-six hectares) et sa localisation en milieu dense et déjà habité, son déroulement dépend de la prise en compte de différentes temporalités individuelles et collectives, représentées par les temps de travail, de consommation et de loisirs des usagers, les mandats politiques (à l'échelle de l'arrondissement et de la Ville), les cycles économiques et les projets qui font l'opération d'aménagement. Ceux-ci sont plus ou moins longs et saccadés, rythmés par la mise en œuvre d'infrastructures, ponctués par des diagnostics, des livrables, des moments de concertation, des phases de travaux et de vacance des lieux. La transformation de l'espace en paysage attractif suppose de bien s'articuler à ces rythmes et ces temporalités pour renverser les représentations négatives dont les chantiers peuvent faire l'objet (nuisances sonore, visuelle, etc.), d'animer les zones de travaux pour gérer l'attente des riverains et susciter la venue d'usagers.

Si le street art est aujourd'hui utilisé pour répondre à de tels enjeux dans des territoires en cours de mutation ${ }^{3}$, c'est parce qu'il est reconnu par les institutions en tant que vecteur de marketing urbain (Mc Auliffe, 2012). Cette reconnaissance est liée au succès de la ville festive et événementielle (Chaudoir, 2007; Gravari-Barbas, 2009), de l'urbanisme temporaire ${ }^{4}$ (Gwiazdzinski, 2009 ; Pradel, 2010), et des préceptes de la ville créative (Florida, 2002) - d'après lesquels l'art et l'événement serviraient au développement économique et social des villes. Ephémère, processuel (Volvey, 2011), réversible, situationnel, contextuel (Ardenne, 2009) et performatif, il sert à nourrir la valeur communicationnelle des espaces publics (Habermas, 1978) en augmentant leur dimension sensible dans un contexte d'économie post-industrielle et de société postmoderne. Nous le préciserons, ses formes s'adaptent et tirent même profit des caractéristiques temporelles, spatiales, et de la présence d'acteurs de l'urbanisme et 
l'aménagement, susceptibles de commander et financer des créations artistiques pour servir l'image de leur activité.

5 Avant d'aller plus loin, il importe de préciser la manière dont le terme de street art est employé dans le cadre de cet article. Paru au début en France des années 1990 et consacré par les médias à la fin des années 2000, le vocable fait l'objet de nombreux débats et regroupe des courants contradictoires. Ces caractéristiques varient en fonction du temps (Blanché, 2015), des acteurs, du contexte socio-économique, politique et culturel, mais aussi du statut juridique et des caractéristiques esthétiques des créations. Nous le considérons ici en tant que pratique autorisée par les autorités publiques. De plus, nous l'opposons au graffiti, qui correspond à des créations réalisées illégalement - dans un cadre dit off (Vivant, 2006) - et qui souffre d'une connotation péjorative de la part des autorités publiques. ${ }^{5}$. Les points communs entre l'ensemble des pratiques sont qu'elles sont éphémères et qu'elles s'inspirent et/ou prennent appui sur les supports de l'espace public. Cette distinction entre street art et graffiti vise à poser un cadre pour examiner les interactions entre le in et le off, tout en sachant que les limites entre les deux sphères sont poreuses. Cette porosité s'explique par le fait que les artistes circulent aujourd'hui librement entre ces sphères Elles se comprennent également par le lien étroit entre l'émergence du street art dans la continuité du graffiti développé en France dans les années 1970.

6 L'utilisation du street art par les institutions culturelles et les médias résulte du processus d'« artification» (Heinich, Shapiro, 2012) dont la pratique a fait l'objet, passant de la sphère du non-artistique à la sphère de l'artistique au début des années 2000. Cette artification découle de plusieurs dynamiques complémentaires, parmi lesquelles le renouvellement du cycle de l'art contemporain par ses marges depuis la deuxième partie du XX ${ }^{\mathrm{e}}$ siècle (Heinich, 1998), l'évolution du marché de l'art, la recherche perpétuelle de nouveaux artistes par les collectionneurs, et la professionnalisation des premières générations de graffeurs devenus artistes contemporains et galeristes.

7 Dans un tel contexte et à partir du cas de Paris Rive Gauche, l'objectif de cet article est, d'un côté, de comprendre de quelle manière le street art s'alimente des caractéristiques des territoires en mutation urbaine pour en nourrir l'image et l'attractivité, et de l'autre, d'identifier les effets de son utilisation sur les modes de présence et de valorisation temporelles de la pratique et son renouvellement par le graffiti dans le temps plus ou moins court. L'ambition est d'examiner, non pas les objets, mais les mécanismes et les hybridations impliqués et suscités par leur valorisation, en portant un intérêt particulier pour les temporalités artistiques et urbaines.

8 Pour répondre à cet objectif, cet article est divisé en deux parties. Une première partie porte d'abord sur l'identification des signaux qui attestent de la reconnaissance du street art en tant qu'agent d'image positive de Paris Rive Gauche, tant par les autorités publiques, que par les acteurs de l'aménagement et de l'art. Elle examine ensuite les manières dont la pratique artistique accompagne et anime les temporalités de la mutation urbaine, en proposant des expériences qui y sont intrinsèquement liées. Une seconde partie analyse les effets du succès institutionnel de la promotion du street art. En premier lieu, elle met en avant le changement de valeur des productions et des dispositifs de valorisation, qui passent de l'éphémère au pérenne, et en interroge certains effets urbains et artistiques. En second lieu, elle s'intéresse aux possibilités de 
renouvellement du street art par le graffiti en montrant que le street art, inspiré du graffiti, attire en retour son augmentation.

Les résultats présentés reposent sur une méthodologie pluridisciplinaire, nourrie par une double approche professionnelle et académique. D'un côté, nous avons adopté une démarche par l'immersion et la participation observante en tant que chargée de mission culturelle à la SEMAPA, société d'aménagement responsable de la ZAC Paris Rive Gauche ${ }^{6}$. De l'autre, nous avons croisé plusieurs techniques d'enquêtes incarnées entre autres par la création d'une base de données des projets de street art réalisés dans la ZAC depuis le démarrage de l'opération et la conduite de vingt-huit entretiens semidirectifs auprès d'acteurs impliqués dans la production et la promotion du street art entre 2013 et 2017. Parmi eux figurent dix artistes qui circulent entre le in et le off, quatre organisateurs de projets de street art, deux acteurs de la mairie du 13 arrondissement, douze professionnels de l'aménagement qui travaillent dans la société d'aménagement, dans des sociétés de promotion, des agences d'architecture et chez des bailleurs sociaux.

\section{Comment le street art se nourrit et alimente l'image de la mutation urbaine}

\section{Production et médiatisation du street art dans Paris Rive Gauche}

Entre 2011 et 2017, plus d'une trentaine d'œuvres de street $\operatorname{art}^{7}$ ont été commandées ou autorisées dans la ZAC, qui fait elle-même partie d'un arrondissement marqué par la promotion institutionnelle du street art depuis la fin des années 2000. Parmi les œuvres figurent dix-huit fresques monumentales peintes sur des façades murales, des installations conçues à l'occasion d'évènements dans des zones de chantier, tant en extérieur (échangeur du périphérique, alcôves autour de la Halle Freyssinet, etc.) qu'en intérieur (appartements d'un bâtiment de logements vacants), et plus d'une quinzaine de collages et de pochoirs sur des éléments du mobilier urbain, tel que des générateurs électriques et potelets anti-stationnement. À ces productions, s'ajoutent des outils, à l'instar du panneau d'exposition «Mur XIII » installé sous la passerelle Simone de Beauvoir depuis 2012, utilisé pour présenter des œuvres temporaires d'artistes. 


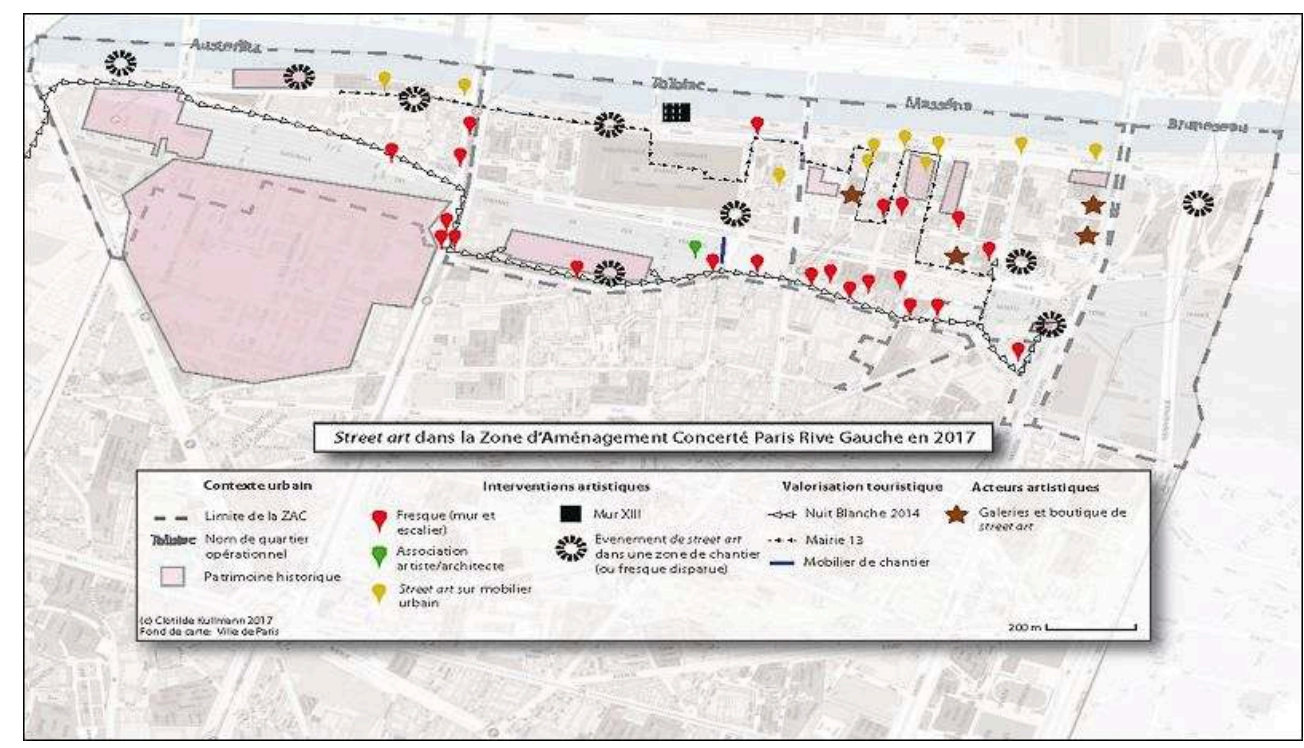

Depuis la fin de l'année 2013, la vitesse d'augmentation du nombre d'interventions s'est considérablement accélérée. Ce phénomène s'explique par le succès de la Tour Paris 13, une exposition dédiée au street art dans et sur un immeuble en attente de démolition, organisée par le directeur de la galerie Itinerrance ${ }^{8}$ en octobre 2013. Cent-huit artistes sont intervenus pour métamorphoser les pièces de trente-six appartements du bâtiment en œuvres immersives et habiller ses façades par des collages. Quant à sa démolition en avril 2014, elle a été transformée en performance artistique lors de son grignotage par des grues, laissant progressivement apparaitre les intérieurs des appartements. La manifestation a attiré un nombre important de visiteurs ${ }^{9}$, a été fortement relayée dans les médias et les mondes de l'art (Becker, 1988) et a transformé l'immeuble en icone artistique temporaire à l'échelle du quartier.

Depuis lors, plusieurs signaux attestent de la reconnaissance, par les autorités publiques, les acteurs de l'aménagement et de l'art, du street art comme vecteur d'image positive et attractive dans Paris Rive Gauche. Ce phénomène s'illustre par l'intérêt grandissant des professionnels de l'aménagement qui financent, commandent, organisent et autorisent la production et la médiatisation du street art dans la ZAC. Les productions sont soutenues par la mairie du $13^{\mathrm{e}}$ arrondissement et coordonnées par les directeurs des galeries de street art ouvertes dans le secteur. Ils font l'intermédiaire entre les acteurs de l'aménagement et de l'art, coordonnent la réalisation technique des interventions, trouvent des financements et utilisent leur carnet d'adresses de galeriste au service des projets en extérieur. La prise d'importance de ces acteurs se vérifie spatialement par le développement d'un nouveau pôle de galeries ${ }^{10}$, qui succède à celui des galeries d'art contemporain de la rue Louise Weiss, dont l'ère de gloire date de la fin des années 1990 et du début des années 2000. De la sorte, la galerie Itinerrance été rejointe par la galerie Mathgoth et la boutique le Lavomatik en 2014.

À titre d'exemple, la SEMAPA a commandé à la galerie Itinerrance une anamorphose de l'artiste 1010 pour couvrir une partie de l'échangeur du boulevard périphérique en 2015 , fermé à la circulation en prévision de sa démolition. Cette création a en partie été financée par l'entreprise de travaux et les bureaux d'études impliqués dans le projet urbain. Un an après, la société d'aménagement a sollicité la galerie Mathgoth pour la réalisation, par l'artiste Mademoiselle Maurice, d'une œuvre composée de quinze mille 
origamis en forme d'oiseaux sur une barre d'immeubles de la ZAC Paul Bourget ${ }^{11}$, quelques mois avant sa destruction.

Le succès de la promotion territoriale par le street art se vérifie par ailleurs par le changement de comportement des acteurs culturels du centre historique de la métropole parisienne vis-à-vis de Paris Rive Gauche et ses galeristes. En témoigne l'organisation dans la ZAC du parcours open musée street art contemporain de la Nuit Blanche 2014, une manifestation à vocation internationale emblématique de la métropolisation par la culture. Ou encore, la coordination, par le directeur de la galerie Itinerrance, de plusieurs projets dans des lieux symboliques du centre historique et culturel de la métropole. En 2015, il a ainsi organisé l'animation artistique des palissades de chantier du Pont des Arts et installé une sphère de Shepard Fairey, alias Obey, au $2^{\mathrm{e}}$ étage de la Tour Eiffel, qui représente Paris à l'international.

En plus de ces circulations et sollicitations, on note une multiplication du nombre d'expositions dans des endroits en mutation depuis 2013. Elles sont organisées selon des modalités qui rappellent celles de la Tour Paris 13 et par des acteurs aux profils de plus en plus diversifiés. Les lieux appartiennent à des bailleurs, des promoteurs, des municipalités et même à des particuliers, rassurés et séduits par le succès et la maitrise de la Tour Paris 13 (Échange informel avec un bailleur, 2014). Ils sont mis en œuvre par des figures intermédiaires qui disposent là encore de compétences à la croisée des champs entre l'art et l'urbain. Ils ont une formation et/ou une expérience en tant qu'urbanistes, designers, artistes, galeristes. Ils interviennent individuellement ou sont organisés en association, en collectif et en entreprise. Par exemple, le directeur de l'association Art en Ville, qui a travaillé pendant plusieurs années en tant qu'urbaniste, a été engagé en 2014 par la société d'aménagement Grand Paris Aménagement pour faire intervenir une quarantaine d'artistes lors du festival In Situ Art Festival dans la ZAC du Fort d'Aubervilliers, avant le démarrage des travaux. Dans un autre cas, l'association d'artistes INZOUK ASSOC a organisé l'exposition Le Grand 8 avec une cinquantaine d'artistes dans un hangar de Malakoff en 2016. Le lieu était en attente de démolition et appartenait cette fois à une personne privée. Forte de son succès, la même association a monté, en 2016, l'exposition $L A B 14$ avec une trentaine d'artistes, dans un immeuble de la Poste du $14^{e}$ arrondissement de Paris avant sa réhabilitation. La même année, les parties intérieures du bâtiment de la Maison des Arts et Métiers étaient investies par quatre-vingts artistes pour l'exposition Rehab 2, orchestrée par une l'association d'artistes Bitume pendant une période de travaux.

La multiplication des productions du street art dans la ZAC et d'autres endroits en mutation, qu'ils soient en périphérie ou dans le centre historique de la métropole parisienne, ainsi que les circulations des acteurs entre le centre métropolitain et Paris Rive Gauche, prouvent que le street art est légitimé en tant qu'ingrédient de la formation d'une image positive et attractive par les acteurs de l'aménagement, de l'art, voire de la société civile, pendant des périodes de mutation urbaine. Il convient dès lors de comprendre la manière dont les interventions s'appuient sur les caractéristiques de la mutation pour servir l'image de la fabrique urbaine. 


\section{Des offres temporaires qui s'appuient sur l'expérience de la mutation urbaine}

Si le street art est considéré comme porteur d'image positive dans des endroits en mutation, c'est parce que ses dimensions labiles, éphémères et réversibles lui permettent de se synchroniser aisément aux temporalités des opérations d'aménagement. Les œuvres épousent les changements spatiaux, les mouvements des destructions, constructions, dégradations et réhabilitations qui défont et remodèlent sans cesse la ville.

Certaines fresques monumentales sont conçues dans l'objectif de conférer à l'architecture des Trente Glorieuses une valeur esthétique nouvelle dans le temps long, comme le Baptême de Borondo situé sur une façade de la rue du Chevaleret depuis la Nuit Blanche 2014. Leur accumulation au fil du temps contribue à la constitution de collections permanentes dans l'espace (Boltanski et Esquerre, 2017). La ligne 6 du métro, dont le tronçon est aérien entre les stations Bercy et Place d'Italie, tout comme la rue du Chevaleret, sont aujourd'hui bordées par des fresques monumentales qui tendent à former un ensemble d'œuvres cohérent et complémentaire.

Illustration 3 - Borondo, Le Baptême, rue du Chevaleret, 2014

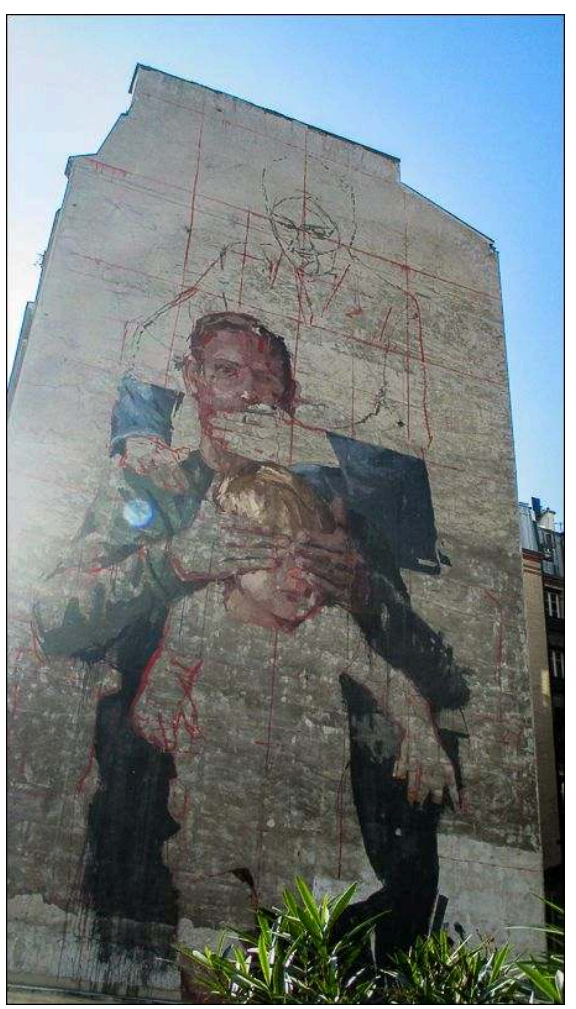

Auteur : C. Kullmann.

D'autres sont au contraire commandées spécifiquement en amont de processus de démolition ou de construction afin d'animer temporairement les espaces publics, à l'instar de l'Hommage à Farhat Hached, réalisée par l'artiste Dabro sur une façade de l'avenue de France en 2013, avant d'être cachée par l'édification d'un nouveau bâtiment deux ans plus tard. 
Ces créations peuvent être programmées de manière ponctuelle ou répétée dans le temps sur un même support de manière à rythmer l'animation et renouveler l'image des espaces publics. En témoigne le M.U.R XIII sur lequel est présenté une nouvelle œuvre tous les quinze jours ou tous les mois, et les générateurs électriques régulièrement ré-habillés par une nouvelle production.

Les modes de valorisation in et ex situ jouent donc eux-mêmes sur les ambiances et s'appuient sur les dispositifs de la mutation urbaine. De manière non exhaustive, la mairie du $13^{\mathrm{e}}$ arrondissement a mis en ligne six parcours de " randonnée " sur son site Internet ${ }^{12}$ en octobre 2013. La même année, la SEMAPA a installé une palissade de chantier d'une longueur de quatre-vingt-huit mètres, faisant apparaître des photographies des œuvres et un plan de leur localisation. Cette plaquette touristique in situ se trouve sur une nouvelle place Jean Michel Basquiat, une figure emblématique du graffiti. Les offres proposées s'inscrivent dans le contexte du succès de l'exploration urbaine $^{13}$ et des offres touristiques qui jouent sur la mise en scène des lieux ordinaires et les expériences décalées et originales (Condevaux, Djament-Tran et Gravari Barbas, 2016). Greg Richard et Julie Wilson l'ont souligné, la concurrence croissante entre les villes «signifie que « les biens et les services ne sont plus suffisants et que les producteurs doivent différencier leurs produits en les transformant en " expériences » qui engagent le consommateur " (Richard et Wilson, 2006, p.1 210). Les expériences sont immédiates, convoquent l'imagination, font appel au vécu, au subjectif et à l'intuition (Zeitler et Barbier, 2012). C'est ainsi que les visiteurs de la Tour Paris 13 sont allés " chez les autres ». Ils ont accédé à la sphère privée d'inconnus en se rendant dans des appartements dont certains portaient encore les traces de la présence de leurs anciens occupants (papiers peints, mobilier de cuisine et de salle de bain...). Quant à la dimension attractive de la manifestation, elle a été stimulée par l'annonce publique de la démolition du bâtiment dès le lendemain de sa fermeture. Ou alors que les publics de l'exposition le Grand 8 étaient conviés à vivre une expérience ludique en participant à "une attraction artistique, enchantée, fantaisiste» pendant laquelle « le spectateur s'amuse, ressent, joue, contemple avec la lumière, les peintures, les objets, les structures pour lui rappeler des moments de la vie tels que, l'enfance, le rire, l'animal, le végétal et la ville ${ }^{14} »$. 


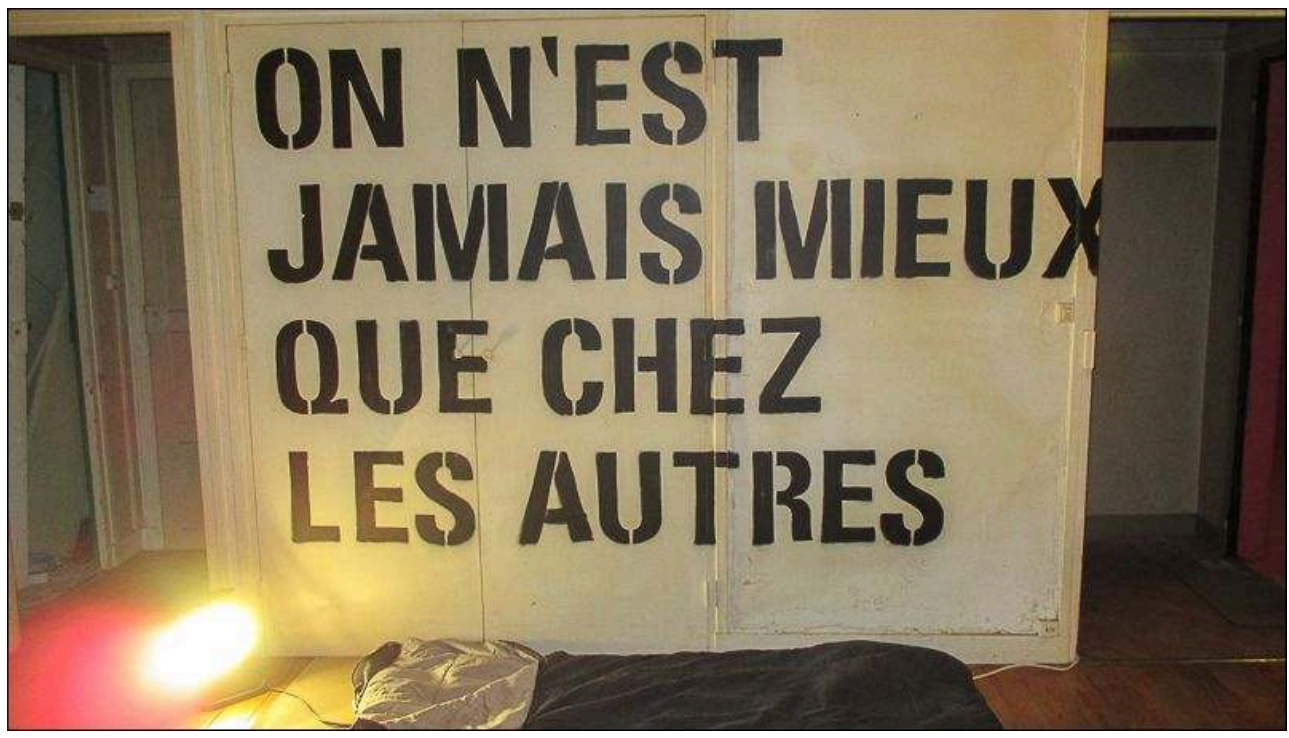

Auteur: Simon Pouillaute.

Illustration 5 - Publics dans l'emprise spatiale de la gare Masséna lors de la Nuit Blanche 2014, Paris $13^{\mathrm{e}}$ arrondissement

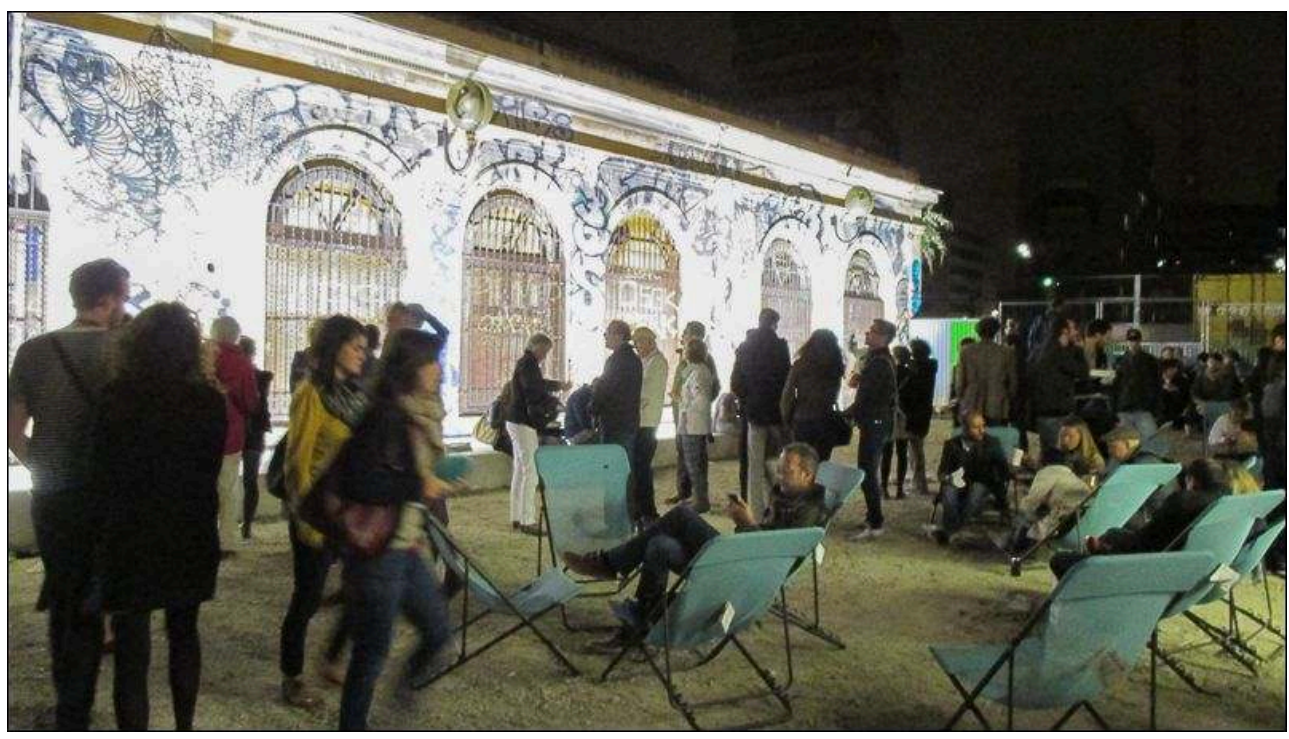

Auteur: C. Kullmann.

22 Dans un autre genre, c'est l'emprise de chantier autour de la Gare Masséna qui a été ouverte et transformée en lieu de détente et de consommation à l'occasion de la Nuit Blanche 2014. Les murs de la Gare avaient été recouverts par des collages de l'artiste Swoon, et ses pourtours, équipés de transats et de foodtrucks.

Les productions et les actions de valorisation du street art ont finalement vocation à esthétiser et rythmer l'animation des espaces urbains, à proposer des ballades touristiques atypiques, à transformer des lieux ordinaires en destinations extraordinaires en générant des expériences qui font appel au sensible et à l'effet de surprise. Ce succès étant en partie fondé sur l'immédiateté, on peut s'interroger sur ses effets dans le temps long. Déjà, on observe des changements de forme de la pratique 
artistique et, dans le temps court, des interactions avec le graffiti susceptibles d'alimenter la reproduction des projets.

\section{Effets sur les temporalités du street art et son renouvellement par le graffiti}

\section{Pérennisation du street art et impacts sur la pratique artistique}

Depuis 2014, on observe les prémices d'un changement de valeur du street art, qui se traduisent par la pérennisation de certaines de ses formes. Ce phénomène s'explique d'abord par la «stabilisation" des constructions et la diminution du nombre de démolitions. Au fur-et-à-mesure de l'avancement d'une opération d'aménagement, le paysage laisse naturellement de moins en moins de place à l'éphémère et à l'expérimentation, le nombre de bâtiments à démolir ne cesse de diminuer et les emprises de travaux disparaissent. De plus, l'éphémère fait le succès de l'art et est porteur de valeur parce qu'il permet de surprendre en " (re)vendant » des espaces à plusieurs reprises (Garnier, 2008). Toutefois, avec la répétition de projets similaires, leur caractère surprenant s'évanouit progressivement, d'autant que la reproduction de mêmes objets à l'infini était appréciée à l'époque fordiste mais a perdu de sa valeur dans la société post-libérale et postmoderne, avec la célébration de la subjectivité.

La prolongation de la durée de vie des œuvres se vérifie par des gestes. Des mesures apparaissent pour la restauration des fresques, alors qu'elle n'avait pas été prévue lors de leur conception. Ainsi, le fond bleu du Chat réalisé par l'artiste français Christian Guémy, alias C215, a été repeint en 2017, quatre ans après sa création. Aussi, des projets qui avaient été commandés spécifiquement pour un évènement ponctuel, sont recyclés pour marquer plus durablement l'espace, à l'instar du pochoir Être étonné c'est un bonheur créé par l'artiste français Jacques Villeglé pour la Nuit Blanche 2014 puis reproduit quelques mois plus tard sur un nouveau mur dans Paris Rive Gauche. Le temporaire devient une étape de la production du pérenne. Il permet de tester ce qui marquera plus durablement le paysage, en fonction du succès des interventions. 
Illustration 6 - C215, le Chat, avant restauration, 2013

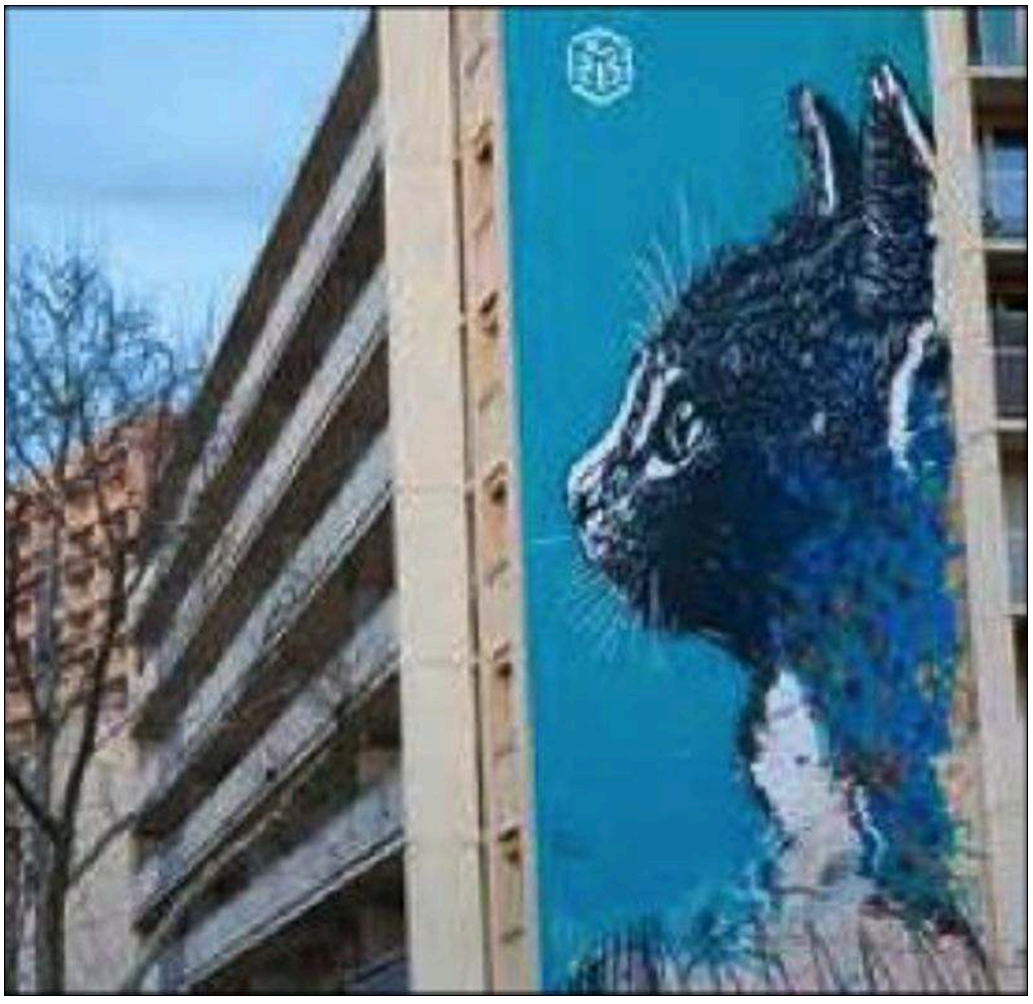

Auteur: C. Kullmann.

Illustration 7 - C215, le Chat, après restauration, 2017

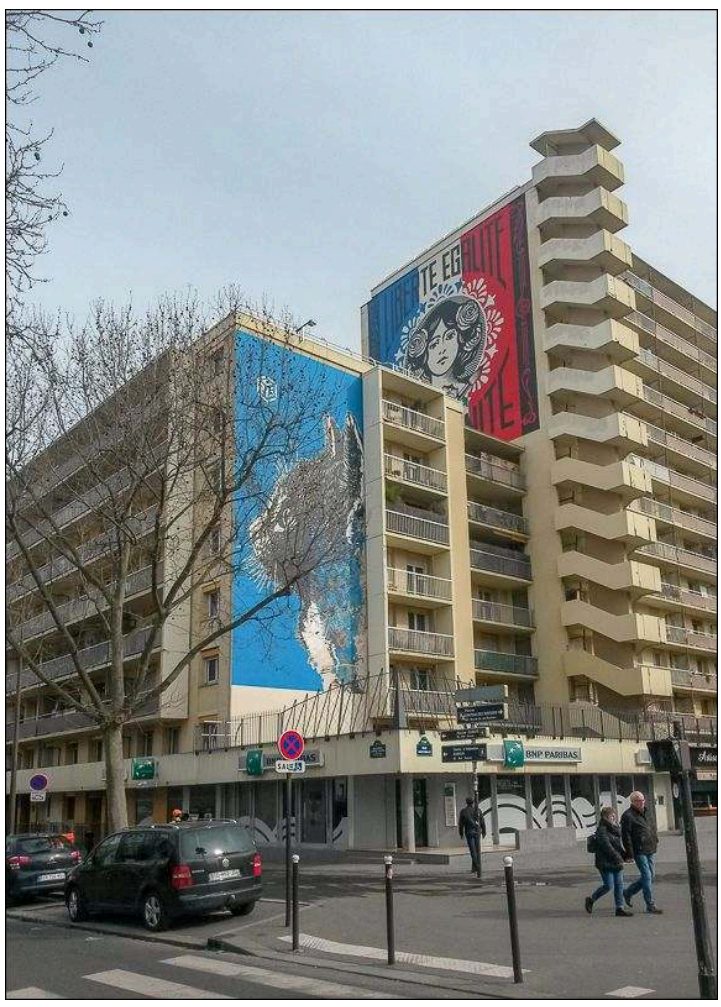

Auteur: C. Kullmann. 


\section{l'exploitation de leur histoire. Cette exploitation se manifeste dans les discours, comme} l'illustre un tweet du Maire du 13 arrondissement représentant la photographie de la fresque « Dégage » de Rero, associée aux mots « déjà 5 ans » en 2017. Ou encore, dans l'envie exprimée par le directeur de la galerie Itinerrance de recréer une ambiance artistique aussi marquante que celle des années folles à Montparnasse au début du $\mathrm{XX}^{\mathrm{e}}$ siècle mais d'en préserver les traces dans le temps long (Entretien, 28/01/2014).

À ces changements in situ s'ajoutent des modes de médiatisation ex situ à travers les dispositifs numériques, qui pérennisent les interventions et participent de leur mise en récit (Boltanski et Esquerre, 2017). Par exemple, les étapes de la Tour Paris 13, représentées principalement par la création des œuvres d'art, l'ouverture aux publics et la démolition du bâtiment, ont été reliées au moyen d'un dispositif transmédia (Lamin, 2014). D'un côté, le projet a été retracé de manière linéaire à travers la réalisation d'un film ${ }^{15}$ encore présenté dans divers festivals en France et dans le monde en 2018. De l'autre, il a été valorisé de manière non linéaire et interactive par la création d'un site Internet ${ }^{16}$ qui conviait les internautes à déambuler dans les salles de l'exposition. premier lieu, la pérennisation de certaines formes de street art pourrait se transformer en patrimonialisation tant du street art que de ses supports architecturaux, sachant que la patrimonialisation est une stratégie de démarcation dans le cadre des projets de régénération urbaine (Guinand, 2015). Des initiatives apparaissent déjà pour rehausser la valeur patrimoniale des lieux promus par le street art, à l'instar de la réouverture du festival Art In Situ Festival lors des Journées du Patrimoine ${ }^{17}$ en septembre 2014. Ces dynamiques interrogent non seulement sur la mémoire, mais aussi sur les modes et les motifs de patrimonialisation de la ville dans les prochaines années. Plusieurs exemples montrent déjà que les critères de conservation des créations reposent sur l'appréciation des visiteurs, notamment à l'aide du numérique qui place les internautes en acteurs de cette patrimonialisation. Un «sauvetage numérique » des œuvres de la Tour Paris 13 a ainsi été proposé aux visiteurs du site Internet du projet lors de la démolition du bâtiment. Il faudra alors s'interroger sur le profil des usagers impliqués ou non dans les décisions de conservation.

En second lieu, les conditions juridiques et financières de la production du street art pourraient être affectées par le changement de valeur de la pratique. Si une durée de conservation minimale des œuvres venait à être imposée dans les contrats de production, elle serait logiquement associée à des obligations d'entretien et donc à des coûts supplémentaires. Or, l'intérêt des municipalités pour les fresques s'explique notamment par leur moindre coût par rapport à un ravalement. Le nombre de fresques continuerait-il alors à augmenter si rapidement dans le temps? De surcroît, les procédures de production des œuvres deviendraient plus contraignantes pour les commanditaires publics. À l'heure actuelle, c'est le caractère éphémère des créations qui permet de se soustraire à certaines contraintes de la commande publique et de solliciter des artistes au gré-à-gré.

En troisième et dernier lieu, alors que la dimension éphémère et le caractère réversible du street art permettent aux artistes de participer aux démarches d'urbanisme temporaire et transitoire, la pérennisation des créations leur offre de nouvelles perspectives de collaboration, entre autres avec les architectes. Dans Paris Rive Gauche, 
les architectes, qui ont depuis 2013 l'obligation de faire appel à des artistes durant la phase des concours de maitrise d'œuvre, se tournent désormais vers les artistes de street art. Pour preuve, l'équipe d'architectes de Fabrice Dusapin a sollicité l'artiste Vhils afin qu'il réalise une sérigraphie pérenne sur des façades en rez-de-chaussée d'un nouveau bâtiment.

31 Tandis que le street art s'apprête à marquer durablement le paysage urbain, le renouvellement des approches dans le temps court est à chercher du côté du graffiti car, en plus d'être inspirée par les codes de ce dernier, la promotion territoriale par le street art provoque indirectement sa multiplication.

\section{Le graffiti comme inspiration et pratique du street art} graffiti venant se superposer par-dessus le street art et ainsi de suite. En atteste l'illustration 8 qui montre une personne en train de graffer par-dessus les fresques des artistes programmés lors de la Tour Paris 13. Selon l'organisateur de l'exposition, il se serait instauré un processus de réappropriation continue des façades entre artistes invités et non invités (Entretien, 28/01/2014). Il en va de même concernant la palissade de chantier portant sur la thématique du street art dans Paris Rive Gauche, régulièrement recouverte par de nouveaux graffitis depuis son installation en 2013. Après avoir remplacé des planches graffées à plusieurs reprises, la SEMAPA a finalement laissé les individus agir, et, un peu par obligation, la palissade vivre avec la ville. Ces mécanismes font écho au jeu stylistique du graffiti et à l'éclosion d'espaces de contestation (Lefebvre, 1974) suscités par la promotion du street art. Ils cristallisent, par des formes et des mots, des volontés de réappropriation et de contrôle d'un même endroit et des tensions entre les artistes invités et non invités dans le cadre du in.

Ensuite, le graffiti se propage autour des interventions et des lieux de diffusion du street art. Ainsi que le montre l'illustration 9, des graffeurs se sont appropriés des escaliers publics inscrits dans le parcours de la Nuit Blanche 2014. Ils interviennent également 
régulièrement sur le promontoire et le mur qui bordent la boutique le Lavomatik, et sous les fresques monumentales.

Illustration 8 - Intervention d'un graffeur sur la Tour Paris 13 en octobre 2013

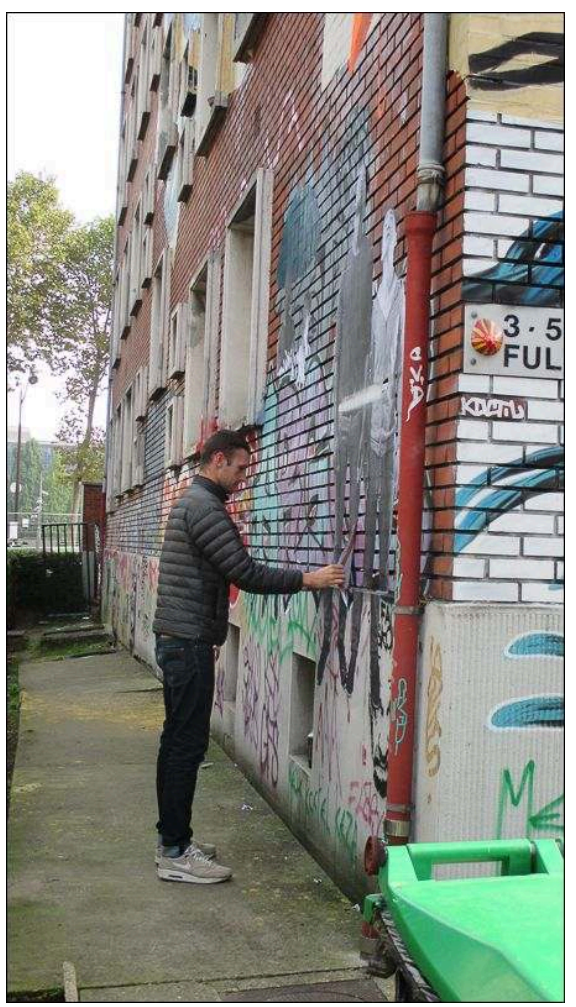

Auteur : C. Kullmann 
Illustration 9- Graffitis réalisés à proximité du Lavomatik, en mai 2017

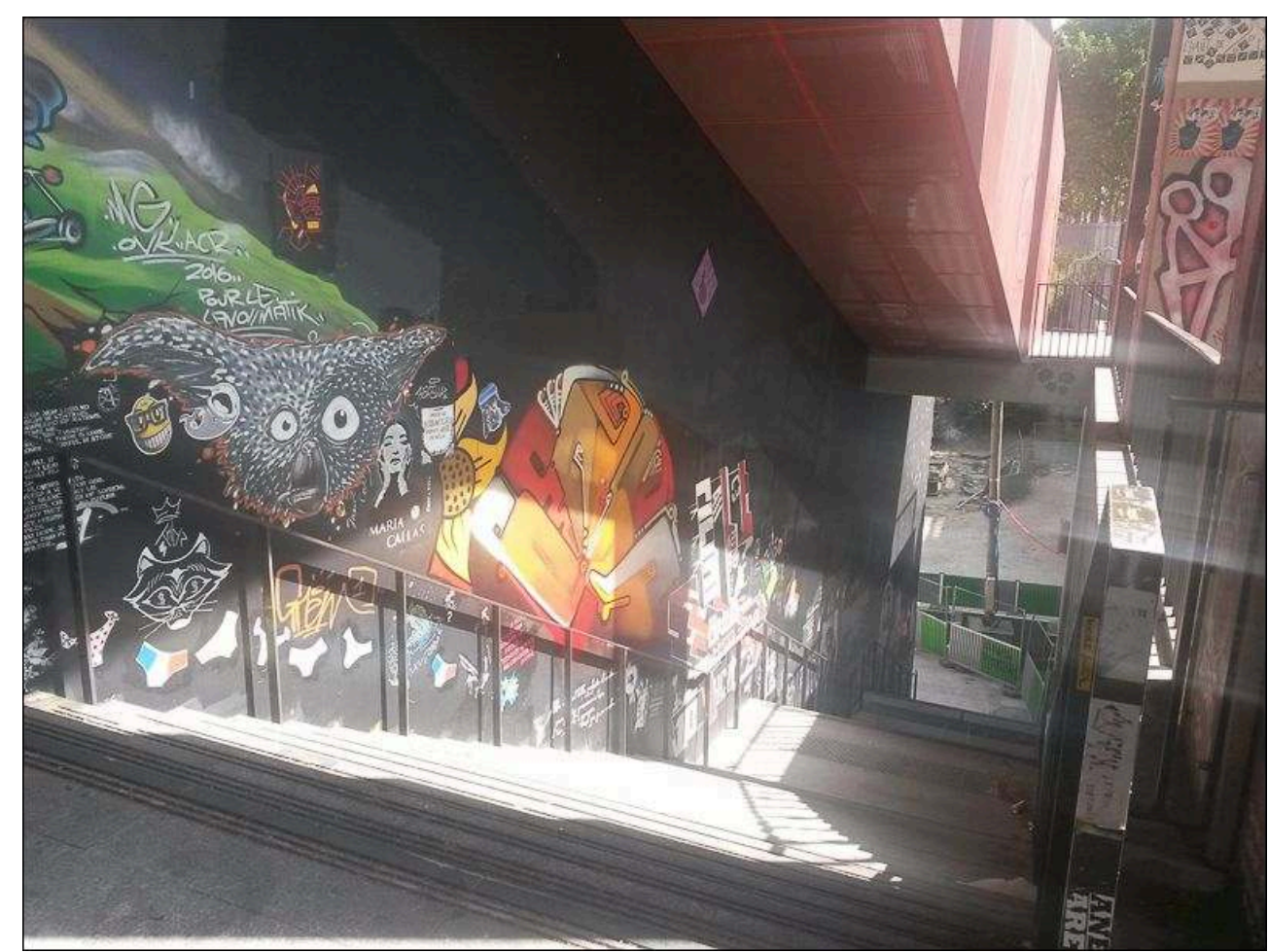

Auteur : C. Kullmann.

37 La capacité du graffiti à renouveler l'image urbaine par le street art, tant en termes de signatures d'artistes que de contenus iconographiques, dépend évidemment de sa visibilité dans le temps, qui résulte elle-même de l'attitude de l'autorité publique dont la mission est d'entretenir les espaces publics. Dans quelle mesure agit-elle en faveur des interactions entre le in et le off? Suit-elle la législation en sanctionnant les interventions illégales ou crée-t-elle son propre règlement? Le cas de l'attitude du Maire $\mathrm{du} 13^{\mathrm{e}}$ arrondissement est emblématique de la souplesse croissante dont les autorités font part vis-à-vis des interventions réalisées hors du cadre législatif. À titre d'illustration, lorsque la RATP a annoncé vouloir intenter un procès à l'artiste M. Chat, pour avoir peint des " chats " ${ }^{18}$ sur un mur dans la station Chatelet du métro parisien en 2014, le Maire a publiquement annoncé qu'il considérait comme injuste cette poursuite en raison de la dimension éphémère du mur alors en travaux. Il a fait connaitre son avis via son compte Twitter : « L'artiste M. Chat poursuivi pour avoir peint dans le métro @leParisien_75. Je lui ai proposé de témoigner à son procès ${ }^{19}$ ». L'artiste n'a finalement pas été condamné par la RATP et un an après le tweet du Maire, une fresque lui a été commandée dans l'arrondissement, alors qu'il y graffait parallèlement. Pour autant, la tolérance n'est pas systématique. L'artiste Jean Rooble nous l'a indiqué à propos de Bordeaux : « il est toujours difficile, voire devenu impossible de peindre dans une ville comme Bordeaux sans autorisation... Ce n'est pas parce-que la ville subventionne des événements qu'elle est plus tolérante avec le graffiti " (Entretien, 01/09/2016).

D'après nos enquêtes, l'acceptation institutionnelle des graffitis dépend d'une combinaison de critères collectifs et individuels. Déjà, compte le positionnement de l'artiste dans les mondes de l'art et sa visibilité sur les réseaux sociaux. Depuis l'internationalisation de l'art contemporain dans les années 1960 (Moulin, Quemin, 
1993) renforcée par la globalisation des marchés de l'art, les artistes qui circulent à l'échelle mondiale, qui sont présents dans les grands festivals et visibles sur les réseaux sociaux ont fondamentalement plus de chances de voir leurs graffitis conservés dans le temps long. Pour preuve, les mosaïques réalisées par Space Invader dans Paris Rive Gauche en 2014 sont encore visibles en 2018. Quant aux collages effectués sur les quais par l'artiste Pantonio en parallèle de sa participation à la Tour Paris 13, ils sont restés visibles pendant toute la durée de l'exposition.

Illustration 10 - Street art réalisé par Pantonio pendant la Tour Paris 13, 2013

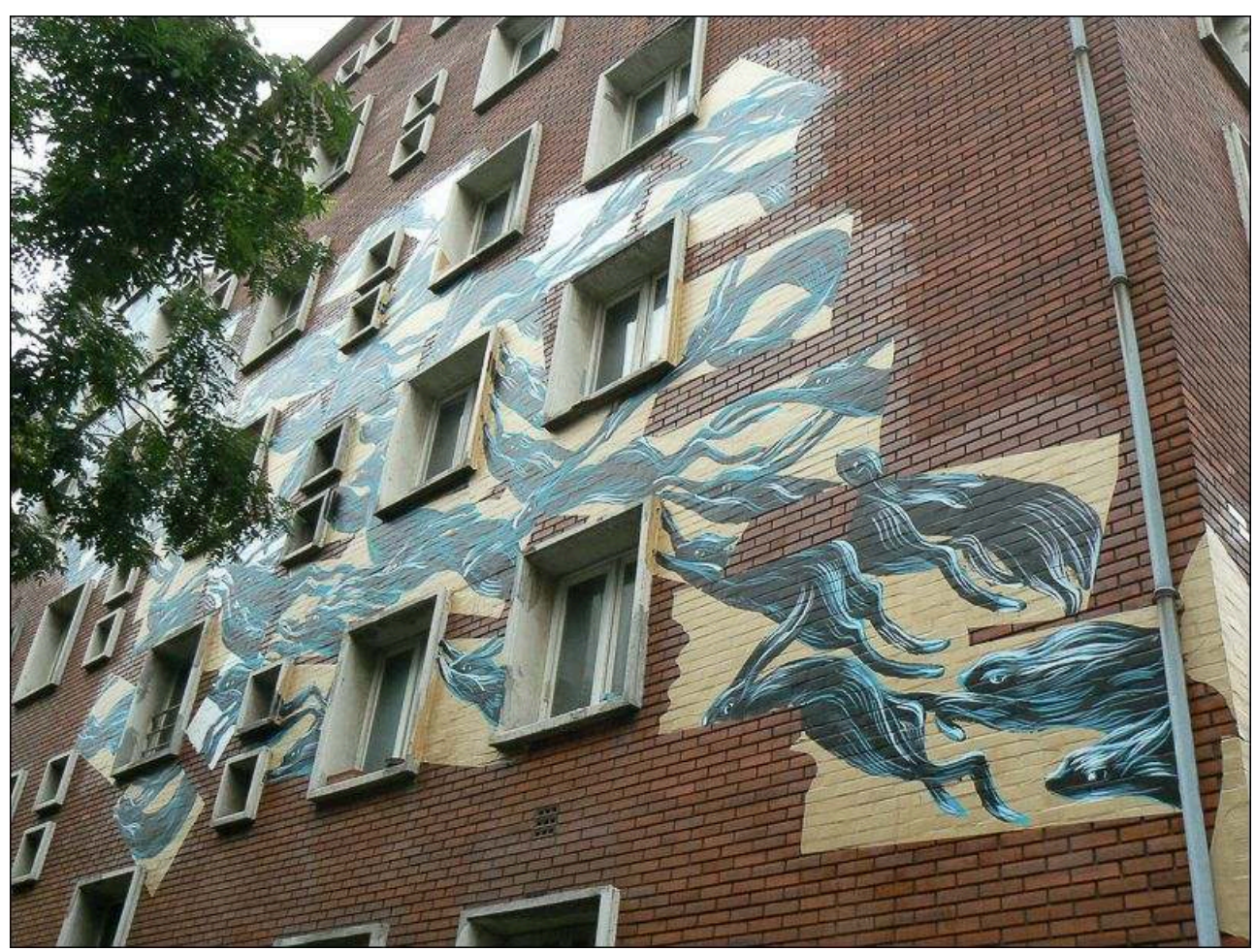

Auteur : C. Kullmann. 
Illustration 11 - Graffiti réalisés a priori par Pantonio pendant la Tour Paris 13, 2013

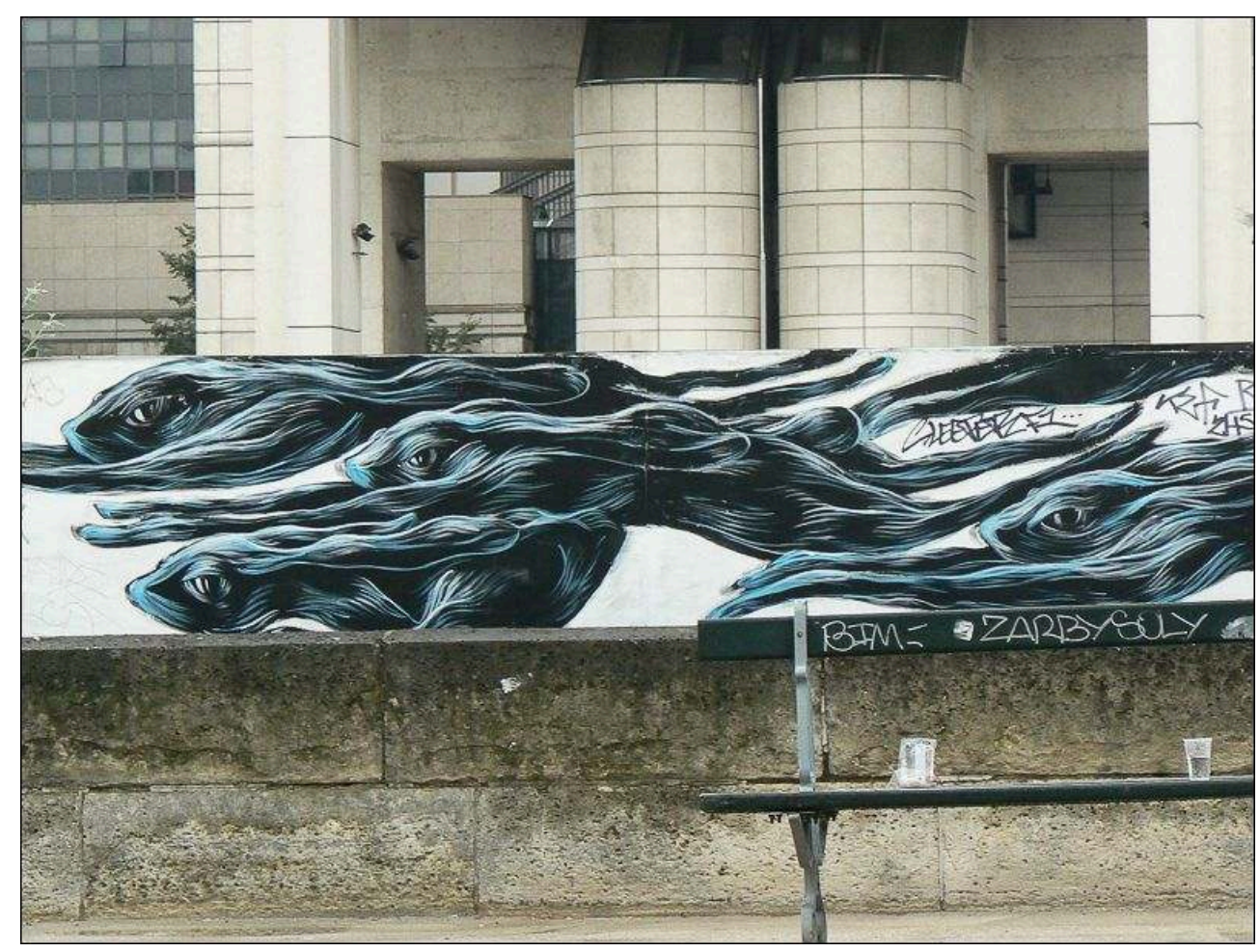

Auteur: C. Kullmann.

39 À ces critères de notoriété s'ajoutent des considérations plus individuelles en lien avec les goûts des autorités, ce qui participe des réflexions sur l'urbanisme postmoderne qui donne une importance particulière au subjectif et à la variété des paysages visuels. En 2014, nous avons ainsi été témoin de la tolérance des services de nettoyage municipaux et de la police vis-à-vis d'un jeune graffeur qui collait une œuvre sur un container à verres, parce que le Maire du $13^{\mathrm{e}}$ arrondissement avait affiché sur les réseaux sociaux qu'il appréciait une œuvre préexistante de l'artiste, sans pour autant connaitre ce dernier.

40 Ces considérations sont complexifiées par la délégation du pouvoir d'autorité à des acteurs du territoire reconnus comme experts du street art. Le directeur du Lavomatik gère par exemple lui-même l'entretien des murs à côté de son commerce, depuis qu'il s'est plaint au Maire de l'effacement systématique des graffitis par les services de nettoyage (Entretien, 31/05/2017).

41 En outre, l'appréhension des critères de tolérance et de valorisation du off nécessite de différencier le street art du graffiti, alors même que le off imite parfois les modes de faire du in, brouillant les pistes entre production formelle et informelle de la ville par le street art. Certains graffeurs mettent en scène leurs créations, par exemple en louant des nacelles, pour laisser penser qu'ils s'inscrivent dans le in et ne pas être arrêtés par les autorités publiques. De cette façon, ils disposent de plus de temps et peuvent réaliser des œuvres sur de grands formats (colloque Oxymore, 2016) ${ }^{20}$. Ils gagnent en compétence et prouvent qu'ils sont capables de s'atteler à des formats imposants. Leur légitimité vis-à-vis des institutions grandit et des commandes leurs sont plus facilement confiées (Échanges informels avec un artiste, 2017). 
42 La pérennisation de certaines formes du street art pourrait donc avoir plusieurs conséquences sur la pratique artistique et la ville, en termes de modalités de production et de forme. Elle pourrait aboutir à la rigidification des conditions de réalisation des créations, à l'ouverture de marchés pour les artistes et les figures intermédiaires, et à l'émergence de nouveaux critères de patrimonialisation pendant voire permis par - la mutation urbaine. Le renouvellement des approches dans le temps court résulte en partie de l'effet indirect de la promotion du street art sur le développement du graffiti, qui peut lui-même marquer durablement le paysage selon les critères d'acceptation et de valorisation des autorités publiques. Ces critères sont subjectifs et évoluent avec l'habitude des usagers, les goûts des autorités et les lois du marché de l'art, l'hybridation et la montée en compétences des artistes au fil de leurs collaborations avec les acteurs de l'urbain et leurs circulations entre le in et le off.

\section{Conclusion}

Le street art est utilisé par les autorités publiques, les professionnels de l'aménagement et de la culture en tant que levier de formation d'une image positive dans Paris Rive Gauche, parce qu'il joue avec ses temporalités, ses spatialités et profite de son système d'acteurs. Les œuvres déployées dans des emprises de chantier et des bâtiments de logements vacants ont vocation à transformer l'espace en paysage, à faire venir des usagers dans des endroits peu voire mal regardés. La force des offres tient à la mise en scène de l'envers du décor urbain, à la proposition d'expériences esthétiques immédiates, qui s'inspirent du graffiti et utilisent de manière créative les caractéristiques, voire les contraintes de la mutation urbaine.

La reproduction des projets et les circulations des acteurs témoignent d'une reconnaissance de l'efficacité du street art en tant qu'agent de marketing urbain et d'image positive pendant la mutation urbaine. D'une part, ces dynamiques alimentent la géographie métropolitaine par le street art en créant de nouvelles connexions, parfois polycentriques, entre les territoires valorisés. D'autre part, elles participent du développement d'un nouveau marché pour les artistes et les figures intermédiaires entre les mondes de l'art et de l'urbanisme.

Tous ces processus vont dans le sens de l'établissement d'une norme du street art pendant et pour la mutation urbaine, la question étant maintenant d'évaluer le degré de labilité de cette norme. Fortes de leur succès, certaines créations commencent déjà à être protégées, pérennisées et utilisées pour rehausser la valeur patrimoniale des supports architecturaux. L'idée est à la fois de prolonger leur durée de vie, d'exploiter leur histoire et de les intégrer dans un ensemble d'œuvres " cohérent " à travers les espaces afin que toutes se complètent et se diversifient (Keck, 2017).

46 Aussi, le renouvellement des approches dans le temps plus ou moins court résulte des interactions entre le street art et le graffiti. Car si le street art s'inspire du graffiti, il attire en retour la venue des graffeurs. Selon le degré et les critères de tolérance individuels et collectifs, tant des autorités publiques que des personnalités considérées comme expertes, le street art peut finalement constituer un outil d'urbanisme alternatif ou servir d'instrument d'éviction des graffitis et de ceux qui l'apprécient en occupant de plus en plus d'espaces. 

street art. Quelle histoire les œuvres servent-elles à raconter? Les contenus iconographiques sont-ils transgressifs, sachant que la permissivité est une valeur positive, à la fois en art contemporain (Heinich, 1998, 2014) et dans le graffiti ? En outre, puisque les galeristes sont au centre des processus de production, leur action agit-elle sur la « galerification » (Kullmann, 2017) des espaces publics, à savoir, sur leur transformation en espaces de galerie?

\section{BIBLIOGRAPHY}

Ardenne P., 2009. Un art contextuel : Création artistique en milieu urbain, en situation, d'intervention, de participation. Paris, Flammarion, $254 \mathrm{p}$.

Blanché U., 2015. Qu'est-ce que le street art ? Essai et discussion des définitions. Cahiers de narratologie, $\mathrm{n}^{\circ} 29$, http://journals.openedition.org/narratologie/7397

Becker H., 1988. Les mondes de l'art. Paris, Flammarion, 382 p.

Boltanski L., Esquerre A., 2017. Enrichissement: une critique de la marchandise. Paris, Gallimard, $672 \mathrm{p}$.

Bourriaud N., 1998. Esthétique relationnelle. Presses du réel, 128 p.

Chaudoir P., 2007. La ville événementielle : temps de l'éphémère et espace festif. Géocarrefour, vol. 82, $\mathrm{n}^{\circ} 3$, http://geocarrefour.revues.org/2301

Condeveaux A., Djament-Tran G., Gravari-Barbas M., 2016. Avant et après le(s) tourisme(s). Trajectoires des lieux et rôle des acteurs du tourisme « hors des sentiers battus ». Une analyse bibliographique.Via@, 2016-1, n 9, http://viatourismreview.com/fr/2016/10/avantet aprestourisme-analysebiblio/

Florida R.L., 2002. The rise of the creative class: and how it's transforming work, leisure, community and everyday life. New York, Basic Books, 434 p.

Garnier J-P., 2008. Scénographies pour un simulacre : l'espace public réenchanté. Espaces et sociétés, $\mathrm{n}^{\circ} 134$, p. 67-81.

Guerini C., 2015. Le street art, entre institutionnalisation et altérité. Hermès, La Revue, vol. 72, n² 2, p. 103-112.

Gravari-Barbas M., 2009. La « ville festive » ou construire la ville contemporaine par l'événement. Bulletin de l'Association de géographes français, vol. 86, n 3, p. 279-290.

Guinand S., 2015. Régénérer la ville. Patrimoine et politiques d'image à Porto et Marseille. Rennes, Presses Universitaires de Rennes, 276 p.

Guinard P., 2014. Johannesburg. L'art d'inventer une ville. Rennes, Presses universitaires de Rennes, $326 \mathrm{p}$.

Gwiazdzinski L., 2009. Chronotopies - L'événementiel et l'éphémère dans la ville des 24 heures. Bulletin de l'Association de géographes français, vol. 86, $\mathrm{n}^{\circ}$ 3, p. 345-357. 
Habermas J., 1978. L'espace public : archéologie de la publicité comme dimension constitutive de la société bourgeoise. Paris, Payot, $328 \mathrm{p}$.

Heinich N., 1998. Le triple jeu de l'art contemporain: sociologie des arts plastiques. Paris, Minuit, 380 p.

Heinich N., 2014. Le paradigme de l'art contemporain: structures d'une révolution artistique. Paris, Gallimard, $384 \mathrm{p}$.

Heinich N., Shapiro R., Brunet F. (dir.), 2012. De l'artification: enquêtes sur le passage à l'art. Paris, Éditions de l'École des hautes études en sciences sociales, 335 p.

Keck F., Boltanski L., Esquerre A., 2017. Enrichissement : une critique de la marchandise. Gradhiva. Revue d'anthropologie et d'histoire des arts, $\mathrm{n}^{\circ} 25$, p. 266-269.

Kullmann C., 2017. La valorisation du projet urbain par la dimension artistique. Quelles perspectives? Thèse de doctorat, Université Paris 1 Panthéon-Sorbonne, 385 p.

Lamin L., 2014. Tour Paris 13 : le transmédia au service de l'art. Circulation, vol. 4, p. 1-17.

Lefebvre H., 1974. La production de l'espace. L’Homme et la société, vol. 31, n 1, p. 15- 32.

Mc Auliffe C., 2012. Graffiti or street art ? Negotiating the moral geographies of the creative city. Journal of Urban Affairs, vol. 34, n 2, p. 189-206.

Moulin R., Quemin A., 1993. La certification de la valeur de l'art. Experts et expertises. Annales. Économies, Sociétés, Civilisations, vol. 48, n 6, p. 1421-1445.

Pradel B., 2010. Rendez-vous en ville ! Urbanisme temporaire et urbanité événementielle : les nouveaux rythmes collectifs. Thèse de doctorat, Université Paris-Est Créteil Val-de-Marne, $549 \mathrm{p}$.

Richards G., Wilson J., 2005. Developing creativity in tourist experiences: A solution to the serial reproduction of culture? Tourism Management, vol. 27, n 6, p. 1209-1223.

Vivant E., 2006. Le rôle des pratiques culturelles off dans les dynamiques urbaines. Thèse de doctorat, Université Paris 8-Vincennes Saint-Denis, 420 p.

Volvey A., 2003. Art et spatialités d'après l'œuvre in situ outdoors de Christo et Jeanne-Claude. Thèse de doctorat, Université Paris I Panthéon-Sorbonne.

Zeitler A., Barbier J.-M., 2012. La notion d'expérience, entre langage savant et langage ordinaire. Recherche et formation, $\mathrm{n}^{\circ}$ 70, p. 107-118.

\section{NOTES}

1. Les ZAC sont des opérations publiques d'aménagement créées par la Loi d'Orientation Foncière du 30 décembre 1967 au sens de l'article L. 300-1 du code de l'urbanisme. Elles ont pour objectif de permettre à une collectivité publique d'engager une opération d'aménagement de l'espace urbain de grande envergure.

2. La Ville de paris et l'APUR (Atelier parisien d'urbanisme) avaient estimé en 1991 que l'opération d'aménagement s'étendrait sur une trentaine d'années. Or, en 2017, cette estimation a atteint une quarantaine d'années.

3. Ces territoires font l'objet de mouvements temporels et spatiaux importants et de bouleversements paysagers, résultants de processus de démolition et de construction, ou encore des (dés)installations de chantiers, etc. dans le cadre d'opérations d'aménagement.

4. Le nombre de friches refonctionnarisées à un titre récréatif et ouvertes temporairement aux publics pendant le temps de vacance des opérations d'aménagement ne cesse d'augmenter, comme le montrent les exemples suivants en Île-de-France en 2017 : La ferme du bonheur (92), le 
Mobilab et la fabrique à rêves au 6b (93), le Ground Control (Paris $12^{\mathrm{e}}$ ), les Grands Voisins (Paris $14^{\mathrm{e}}$ ), le Hasard Ludique et la Gare à Mines (Paris $18^{\mathrm{e}}$ ), etc.

5. Dans les années 1980, l'utilisation du terme de " graffiti » avait déjà une connotation péjorative et justifiait alors des politiques d'effacement et de disqualification par les autorités publiques.

6. Observation conduite dans le cadre d'un doctorat CIFRE (Convention Industrielle par la Formation et la Recherche)

7. Étant donné le caractère rapidement évolutif des œuvres réalisées sur des éléments de mobilier urbain, leur quantité est approximative. Ils n'ont donc pas été inclus dans le tableau.

8. La galerie Itinerrance a ouvert ses portes dans Paris Rive Gauche en 2004.

9. Le nombre de visiteurs varie en fonction des sources médiatiques. BFM TV et Le Figaro indiquaient à la fin du mois d'octobre que 15000 personnes s'étaient rendues à l'exposition (sources : http://www.bfmtv.com/culture/tour-paris-13-temple-ephemere-street-art-fermeportes-635998.html et http://www.lefigaro.fr/arts-expositions/ 2014/04/08/03015-20140408ARTFIG00146-en-direct-la-demolition-de-la-tour-paris-13.php, tandis que le site Internet $\mathrm{du}$ Club Artistic Rezo en comptabilisait 25000 (source: http:// www.artistikrezo.com/2013110414391/actualites/street-art/tour-paris-13-laventure-digitale-

continue.html). La galerie Itinerrance avait calculé, elle, que 30000 visiteurs s'étaient rendus dans le bâtiment (http://itinerrance.fr/tour-paris-13-le-film/).

10. Ces professionnels profitent des opportunités de l'opération urbaine en ce sens qu'ils ont accès à des locaux vacants et bénéficient de loyers moins chers que dans les centralités artistiques historiques de Paris, en particulier dans le quartier du Marais.

11. La ZAC Paul Bourget est située à proximité de Paris Rive Gauche dans le $13^{\mathrm{e}}$ arrondissement et est également aménagée par la SEMAPA.

12. Source : http://www.mairie13.paris.fr/mairie13/jsp/site/Portal.jsp? document_id=16892\&portlet_id=3089\&comment=1\&current_page_id=712 (décembre 2013).

13. L'exploration urbaine est une pratique de plus en plus appréciée par les publics, qui consiste en la pratique d'espaces urbains en friche ou parfaitement fonctionnels mais non accessibles et désinvestis, parmi lesquels figurent les toits, les tunnels et les zones de chantier.

14. Source: http://www.expo-legrand8.com/ (janvier 2017).

15. Le film Tour Paris 13 a été réalisé par Thomas Lallier et présenté dans divers festivals dans le monde après avoir été diffusé sur France ô.

16. Site Internet : www.tourparis13.fr

17. Les journées du Patrimoine ont lieu le troisième week-end de septembre. Pendant deux jours, les hauts lieux patrimoniaux sont ouverts gratuitement au public.

18. M. Chat dessine des chats jaune et orange à la peinture acrylique sur les supports visibles depuis les espaces publics.

19. Source: http://lelab.europe1.fr/Le-maire-PS-du-13eme-arrondissement-de-Paris-JeromeCoumet-soutient-le-graffeur-M-Chat-attaque-par-la-RATP-16222 (janvier 2017).

20. Propos entendu lors du colloque « État de l'art urbain », Oxymore III en 2016.

\section{ABSTRACTS}

The purpose of this article is to analyse the processes and some effects of the production and the valorisation of street art within urban regeneration, in the basis on a development operation in 
the Parisian metropolis (Zone d'Amenagement Concertée Paris Rive Gauche). A first part highlights the signals of the recognition of street art as a tool to create a positive territorial image, by urban and artistic actors. It shows then how the artistic practice is inspired by the characteristics and the experience of the urban regeneration. A second part focuses on some effects of the success of street art. On the one hand, it examines its temporal evolutions, turning from ephemeral to perennial forms, and questions the impacts of these evolutions. On the other hand, it studies the increase of graffitis, which could enrich the urban image by street art according to the criteria of acceptability of the public authorities.

Cet article propose d'étudier les modalités et certains effets de la production et de la valorisation du street art, en tant que pratique légale et encadrée par les autorités publiques, pour valoriser l'image des territoires en mutation, à partir du cas de la Zone d'Aménagement Concerté Paris Rive Gauche dans le $13^{\mathrm{e}}$ arrondissement de Paris. Une première partie porte sur les signaux de la reconnaissance du street art en tant qu'agent de formation d'une image urbaine positive, par les acteurs de l'aménagement et de l'art, puis montre comment la pratique s'alimente des caractéristiques et de l'expérience de la mutation urbaine pour générer cette image. Une seconde partie s'attache à plusieurs effets du succès du street art dans le temps plus ou moins long. D'une part, elle met en avant une pérennisation des formes de la pratique artistique et interroge ses impacts sur les modalités des productions. D’autre part, elle démontre que la promotion du street art attire en retour le graffiti, susceptible d'enrichir l'image urbaine selon les critères d'acceptation des autorités publiques.

\section{INDEX}

Keywords: street art, graffiti, temporality, urban regeneration, experience, ephemeral, perennial

Mots-clés: street art, graffiti, image, temporalité, mutation urbaine, expérience, éphémère, pérenne

\section{AUTHOR}

\section{CLOTILDE KULMANN}

Clotilde Kulmann, clotilde.kullmann@u-pem.fr, docteure en géographie, chargée d'animation scientifique de la Chaire "Aménager le Grand Paris", rattachée à l'Université Paris Est Marne la Valléee. Elle est Chercheuse associée à l'EIREST et a publié récemment :

- Kullmann C., 2017. Street art events and their impact on urban redevelopment. Case study of Paris 13 Tower exhibition in the Paris Rive Gauche Mixed Development Zone. In Murzyn-Kupisz M., Dzialek J. (dir.), The Impact of Artists on Contemporary Urban Development in Europe, Springer. p. 225-240.

- Kullmann C., Bouhaddou M.-K., 2017. In-between new figures of art and urban transformation projects. A French perspective. Articulo - Journal of Urban Research [Online], 15.

- Kullmann C., 2017. La valorisation du projet urbain par la dimension artistique. Quelles perspectives?, Thèse de doctorat, Université Paris 1 Panthéon-Sorbonne, 385 p. 